\title{
Universidad y Sociedad
}

\section{LA PROYECCION SOCIAL EN LA FORMACION PROFESIONAL DEL FISIOTERAPEUTA}

\author{
José Rubén Castillo * \\ José Raúl García * \\ Diana Lucía Marín **
}

\section{Objetivo}

Generar la reflexión sobre la necesidad y las posibilidades de orientar la formación profesional del fisioterapeuta con una perspectiva social.

\section{Metodología}

Este documento desarrolla su temática desde lo general hasta lo particular. Se orienta mediante la elaboración de análisis progresivos que van desde el tratamiento de aquellos aspectos que tienen que ver con la función social de la Fisioterapia, hasta los que tienen que ver con las experiencias que han surgido de la implementación de un currículo en donde tiene presencia la proyección comunitaria como un área especifica de formación.

El contenido se puede diferenciar en cuatro aspectos:

1. La función social de la Fisioterapia

2. Las implicaciones del trabajo comunitario

3. La propuesta de operacionalización curricular

4. La reflexión sobre las experiencias obtenidas, las conclusiones a las cuales se ha llegado y las recomendaciones que surgen de todo lo anterior

\section{DESCRIPCION DE LOS CONTENIDOS}

\section{La función social de la fisioterapia}

El punto de partida del documento es abordar lo relacionado con lo que puede ser la formación académica en las facultades de Fisioterapia, teniendo en cuenta que el elemento básico en todo proceso educativo son las personas que participan en él, con todo aquello que hace parte de ellas, es decir, con sus biografias. También examina la función social de esta disciplina, asumiento que 
ejerce desde las ciencias de la salud y que tiene muchas posibilidades de ampliar su radio de acción, en la medida en que reflexione sobre las concepciones que han orientado su práctica y se decida a recrear su competencia social aprovechando las posibilidades que le brinda la rehabilitación basada en la comunidad. (R.B.C.).

Esta temática tiene respaldo en el análisis que se hace del proceso evolutivo de la concepción de la rehabilitación, de la definición de las politicas y estrategias de atención primaria de salud a nivel internacional y nacional y de las condiciones sociales que se han venido generando en los últimos tiempos, a partir de la de la implementación de la descentralización administrativa y de las cuales surge la necesidad de que el ejercicio profesional del fisioterapeuta se contextúe históricamente de tal manera que le permita responder a los retos que le presenta esta nueva realidad.

\section{Las implicaciones del trabajo comunitario}

Si las acciones sociale se orientan hacia la generación del proceso comunitario, se deduce la necesidad de establecer algunos criterios básicos que permitan identificar la comunidad como una realidad histórica concreta. Se trata de hacer énfasis más en lo práctico que en lo teórico, por lo tanto, al abordarla como un objeto de intervención, se intenta mostrar cómo se presenta y las condiciones en que se construye.

En esta parte se hace una disquisición sobre lo que representa el trabajo comunitario y las implicaciones que tiene; se intenta precisar y distinguir algunos componentes de la comunidad; aquellos que hacen que sea (estructura), las condiciones en que existe (infraestructura); las cuales se diferencian como conceptos en este caso con el fin de señalar aspectos esenciales del trabajo comunitario, aunque somos concientes de que en la realidad tienen nexos, que impiden separarlos.

Al final de esta parte se presentan algunos principios que deben orientar la acción basada en la comunidad teniendo como premisa el reconocimiento explicito de que ante todo cualquier labor comunitaria debe tener de referencia el desarrollo humano de las personas que tienen que ver con ella.

\section{La propuesta curricu- lar}

La implementación de los principios expresados en el segundo punto, condujeron a la necesidad de presentar una propuesta curricularmediante lacual se les de aplicabilidad y no se queden en simples buenas intenciones. Se buscó con ella construir un modelo educativo en el campo de la Fisioterapia, que permita formar profesionales competentes técnica y humanamente para que puedan desempeñarse en nuestro medio.

Por tal efecto, se planteó una concepción pedagógica del Area de Proyección Comunitaria que se enmarca en las posibilidades de las personas que hacen parte del proceso educativo, como complemento se presentó la forma lógica de orientar estos procesos, de tal manera que podamos ser coherentes con los criterios que se presentan a lo largo de éste trabajo. No sobra indicar que la metodología de la propuesta se ve complementada con la estructura general de los contenidos.

\section{Las experiencias}

Tradicionalmente las propuestas aparecen como el punto de partida de ciertas vivencias, en este caso, la propuesta es el resultado de los procesos que se han construido a partir de 
las experiencias que se han promovido en el Area de Proyección Comunitaria de la Facultad de Fisioterapia de la Universidad Autónoma de Manizales

Lo anterior indica que los logros a los cuales se ha llegado son transitorios, puesto que la dinámica de este proceso nos lleva a entender que los resultados son provisonales y que por lo tanto hay mucho por construir.

Por estas razones, vimos la necesidad de plasmar de manera general el sen- tido en que se han desarrollado los procesos de formación académica en proyección comunitaria en la Facultad, los resultados obtenidos y las perspectivas con las cuales se puede orientar la continuidad de estas vivencias. 